\title{
Assessment of Saxagliptin Efficacy: Meta-Analysis of 14 Phase 2 and 3 Clinical Trials
}

\author{
Mikaela Sjöstrand · Cheryl Wei · William Cook · Kristina Johnsson • \\ Pia S. Pollack · Christina Stahre · Boaz Hirshberg
}

Received: January 11, 2017 / Published online: April 21, 2017

(C) The Author(s) 2017. This article is an open access publication

\section{ABSTRACT}

Introduction: This meta-analysis of data from 14 phase 2 and 3, double-blind, randomized, controlled 12 - and 24-week studies $(N=4632)$ summarizes saxagliptin efficacy in patients with type 2 diabetes (T2D) across treatment regimens.

Methods: Patients received saxagliptin $5 \mathrm{mg} / \mathrm{d}$ or control as either monotherapy ( $n=1196$ vs placebo), add-on therapy ( $n=2139$ vs placebo and $n=514$ vs uptitrated sulfonylurea), or initial combination therapy $(n=619$ vs control monotherapy). Patients with renal impairment received saxagliptin $2.5 \mathrm{mg} / \mathrm{d}$ or placebo $(n=$ 164).

Enhanced content To view enhanced content for this article go to http://www.medengine.com/Redeem/ 1818F0601962B3CC.

Electronic supplementary material The online version of this article (doi:10.1007/s13300-017-0261-8) contains supplementary material, which is available to authorized users.

M. Sjöstrand $(\varangle) \cdot$ K. Johnsson · C. Stahre

AstraZeneca, Gothenburg, Sweden

e-mail: mikaela.sjostrand@astrazeneca.com

C. Wei · P. S. Pollack

AstraZeneca, Gaithersburg, MD, USA

W. Cook · B. Hirshberg

MedImmune, Gaithersburg, MD, USA
Results: Mean baseline glycated hemoglobin (A1C) ranged from $8.07 \%$ to $9.43 \%$ for the saxagliptin and control groups across treatment regimens. A1C reduction from baseline was greater with saxagliptin versus control for all studies combined (mean treatment difference [95\% CI]: $-0.55 \%[-0.63 \%,-0.47 \%])$ and when used as monotherapy $(-0.52 \%[-0.63,-0.40 \%])$, add-on $(-0.55 \%[-0.69 \%,-0.40 \%]$ vs placebo; $-0.72 \% \quad[-0.88 \%,-0.56 \%]$ vs uptitrated sulfonylurea), initial combination therapy $(-0.54 \%$ $[-0.73 \%,-0.35 \%]$ vs control monotherapy), and in patients with renal impairment $(-0.42 \%$ $[-0.75 \%,-0.09 \%])$. Similar reductions in A1C versus control were noted for patients $<65$ years $(-0.55 \% \quad[-0.67 \%,-0.43 \%])$ and $\geq 65$ years $(-0.54 \%[-0.69 \%,-0.38 \%])$ and for men $(-0.54 \%$ $[-0.69 \%,-0.40 \%])$ and women $(-0.55 \%$ $[-0.64 \%,-0.47 \%])$ across treatment regimens. More patients achieved A1C $<7 \%$ (39\% vs $23 \%)$ and $\mathrm{A} 1 \mathrm{C} \leq 6.5 \%$ ( $24 \%$ vs $14 \%)$ with saxagliptin than with placebo or active-control treatment. Saxagliptin versus control was associated with a reduction in glucagon area under the curve (AUC) from baseline and increases in insulin AUC, C-peptide AUC, and the homeostasis model assessment of $\beta$-cell function.

Conclusion: Results of this meta-analysis demonstrate the consistency of saxagliptin efficacy in different subgroups of patients with T2D across treatment regimens.

Funding: AstraZeneca. 
Keywords: Dipeptidyl peptidase-4 (DPP-4) inhibitor; Incretin enhancer; Meta-analysis; Saxagliptin; Type 2 diabetes

\section{INTRODUCTION}

Diabetes is a global health problem, and although recent US trends suggest that the prevalence of diabetes has stabilized [1], global projections indicate that the number of individuals with diabetes is set to grow substantially in the coming years [2]. Globally, in 2015, 415 million adults aged $20-79$ years had diabetes, and by 2040 that number is expected to increase to 642 million [3]. Regional increases in diabetes prevalence of 19 to $141 \%$ are expected among adults in Europe (19\%), North America and the Caribbean (37\%), the Western Pacific (41\%), South and Central America (65\%), South-East Asia (79\%), the Middle East and North Africa (104\%), and Africa (141\%) [3]. The prevalence of diabetes is expected to increase across all income levels and age groups [2].

The progressive nature of type 2 diabetes (T2D) [4] necessitates the use of more than one glucose-lowering agent in many individuals with diabetes in order to achieve glycemic control [5]. Current diabetes treatment guidelines recommend an individualized stepwise approach with sequential addition of single oral glucose-lowering agents for patients who do not achieve their glycemic goal within 3 months $[3,5,6]$. The selection of a glucose-lowering agent should take into consideration numerous factors such as efficacy, potential side effects, weight profile, hypoglycemia risk, cost, patient preference, and comorbidities $[5,6]$. Of these factors, comorbidities associated with diabetes such as hypercholesterolemia, hypertension, and obesity are associated with marked morbidity and mortality among patients [7]. Among patients with T2D, the prevalence is $\geq 60 \%$ for each of elevated low-density lipoprotein (LDL), hypertension, and obesity [7]. In addition to increased cardiovascular (CV) morbidity, CV disease (CVD) is a leading cause of death in patients with diabetes and accounts for $>50 \%$ of deaths in patients with T2D [8]. Mortality from heart disease is 2-4 times greater for patients with T2D compared to patients without diabetes [7]. The consequences of diabetes comorbidities are grave, yet the achievement of glycemic, blood pressure, and lipid treatment goals remains suboptimal in patients with T2D, especially in some minority groups (e.g., Mexican Americans and non-Hispanic blacks) [9].

Intensive glucose control early in the course of T2D has been associated with reductions in microvascular complications [10, 11], and, over the long-term, risk reductions in some macrovascular complications have also been reported $[11,12]$. The results of large CV outcomes trials for newer classes of glucose-lowering agents have shown a CV benefit (empagliflozin [13], a sodium-glucose cotransporter-2 inhibitor; liraglutide [14] and semaglutide [15], glucagon-like peptide-1 (GLP-1) receptor agonists) or no increased CV risk (lixisenatide [16], a GLP-1 receptor agonist; alogliptin [17], sitagliptin [18], and saxagliptin [19], dipeptidyl peptidase-4 (DPP-4) inhibitors). Although no evidence of increased CV harm (neutral results for composite end points of major CV events and extended major CV events, including hospitalization for heart failure) has been observed, an increase in hospitalization for heart failure was observed in the $\mathrm{CV}$ outcomes trial for saxagliptin (the Saxagliptin Assessment of Vascular Outcomes Recorded in Patients with Diabetes-Mellitus-Thombolysis in Myocardial Infarction 53 (SAVOR-TIMI 53) trial [19]) and in patients without a history of heart failure in a separate post hoc subanalysis of the CV outcomes trial for alogliptin (Examination of CV Outcomes With Alogliptin Versus Standard Care [17, 20]).

Therapies that have favorable or neutral metabolic effects in addition to glucose-lowering properties are needed. Saxagliptin is an orally active, selective, and competitive DPP-4 inhibitor indicated for the treatment of T2D as an adjunct to diet and exercise to improve glycemic control [21]. Inhibition of DPP-4 prevents the degradation of GLP-1 and glucose-dependent insulinotropic polypeptide (GIP), two gastrointestinal-derived hormones that play major roles in blood glucose control [22]. In individual trials, saxagliptin has demonstrated efficacy and safety as a monotherapy and an add-on combination therapy in patients with T2D [23-29]. Additionally, saxagliptin demonstrated a 
favorable safety and tolerability profile in patients with $\mathrm{T} 2 \mathrm{D} \geq 65$ years of age when saxagliptin was used as a monotherapy or add-on therapy [30-33]. A pooled analysis of 20 randomized controlled studies demonstrated the safety and tolerability of saxagliptin when used as a monotherapy or add-on therapy; however, this pooled analysis did not examine efficacy [34]. The present paper reports a meta-analysis of data from 14 phase 2 and 3, double-blind, randomized, controlled trials that evaluated the efficacy of saxagliptin in relation to glycemic and metabolic end points in patients with T2D across treatment regimens.

\section{METHODS}

\section{Study Design}

This analysis included data from 14 phase 2 and 3, 12- and 24-week, double-blind, randomized, controlled studies $(N=4632)$ examining saxagliptin $5 \mathrm{mg} / \mathrm{d}$ (or $2.5 \mathrm{mg} / \mathrm{d}$ in individuals with moderate [creatinine clearance 30 to $<50 \mathrm{~mL}$ / $\mathrm{min}$ ], severe [creatinine clearance $<30 \mathrm{~mL} / \mathrm{min}$, not receiving dialysis], or end-stage [hemodialysis-dependent] renal impairment) for the treatment of patients with $\mathrm{T} 2 \mathrm{D}$ and inadequate glycemic control (Table 1) [23, 24, 26-28, 35-43]. This analysis included 13 studies from the previously published pooled safety analysis [34] and one more recent study. Seven studies used in the pooled safety analysis were excluded from our meta-analysis because they were either less than 12 weeks in duration, used an active comparator, or they used uptitration of metformin as a control. In the studies included in this meta-analysis, all procedures followed were in accordance with the ethical standards of the responsible committee on human experimentation (institutional and national) and with the Declaration of Helsinki (1964). Informed consent was obtained from all patients before they were included in the studies.

\section{Outcome Measures}

The principal glycemic efficacy end points were mean change in glycated hemoglobin (A1C) from baseline and the percentage of patients achieving A1C targets of $<7 \%$ and $\leq 6.5 \%$. Other glycemic end points that were assessed included mean change from baseline in fasting plasma glucose (FPG), insulin, C-peptide, and glucagon plasma concentrations; area under the curve (AUC) during an oral glucose test for postprandial glucose (PPG), insulin, C-peptide, and glucagon; and mean change from baseline in homeostasis model assessment of $\beta$-cell function (HOMA- $2 \% \beta$ ) and HOMA-2 insulin resistance (IR). Metabolic efficacy end points that were assessed included mean change from baseline in body weight, waist circumference, and body mass index (BMI); mean change from baseline in systolic and diastolic blood pressure; and mean percent change from baseline in fasting lipids (total cholesterol, LDL cholesterol, high-density lipoprotein [HDL] cholesterol, triglycerides, and free fatty acids).

\section{Statistical Analysis}

Baseline was defined as the last assessment on or before the date of the first dose of the double-blind study medication. The difference between saxagliptin and the comparator in change or percentage change from baseline mean $(95 \% \mathrm{CI})$ for each study was determined using an analysis of covariance (ANCOVA) model with treatment group as an effect and baseline value as the covariate. The analyses were performed on the full analysis set, consisting of all randomized patients who had both a baseline and at least one postrandomization measurement included in the analysis. If no end-of-study measurement was available, the last available earlier postbaseline measurement (last observation carried forward, LOCF) method was applied. For patients who started rescue medication before the end of the study, their last postbaseline measurement taken before the date of the first dose of rescue medication was used (prior to rescue). When studies were pooled, the change from baseline mean $(95 \% \mathrm{CI})$ difference between saxagliptin and control for all continuous data glycemic and metabolic end points was determined using Review Manager 5.3 software (RevMan, Cochrane, London, UK). For dichotomous data, the treatment differences in the proportions of patients achieving A1C targets 
Table 1 Trial characteristics

\begin{tabular}{|c|c|c|c|}
\hline Study identifier & Design $^{a}$ & $\begin{array}{l}\text { A1C inclusion } \\
\text { criteria, } \%\end{array}$ & $\begin{array}{l}\text { Patients in } \\
\text { analysis, } n\end{array}$ \\
\hline CV181008 [35] & Monotherapy, treatment-naive ${ }^{b}$ & $6.8-9.7$ & 104 \\
\hline CV181011 [24] & Monotherapy, treatment-naive & $7.0-10.0$ & 195 \\
\hline CV181038 [23] & Monotherapy, treatment-naive & $7.0-10.0$ & 137 \\
\hline CV181063 [36] & Monotherapy, treatment-naive & $7.0-10.0$ & 551 \\
\hline CV181082 [37] & Monotherapy, treatment-naive & $7.0-10.0$ & 209 \\
\hline CV181057 [38] & Add-on to INS+MET & $7.5-11.0$ & 449 \\
\hline CV181014 [39] & Add-on to MET & $7.0-10.0$ & 361 \\
\hline CV181080 [40] & Add-on to $\mathrm{MET}^{\mathrm{b}, \mathrm{c}}$ & $7.0-10.0$ & 158 \\
\hline CV181064 [41] & Add-on to MET & $7.0-10.0$ & 554 \\
\hline CV181117[26] & Add-on to $\mathrm{MET}+\mathrm{SU}$ & $7.0-10.0$ & 254 \\
\hline CV181013 [27] & Add-on to TZD & $7.0-10.5$ & 363 \\
\hline CV181040 [42] & Add-on to SU vs SU & $7.5-10.0$ & 514 \\
\hline CV181039 [28] & Initial combination therapy vs monotherapy & $8.0-12.0$ & 619 \\
\hline CV181062 [43] & $\begin{array}{l}\text { Renal impairment, add-on to existing } \\
\text { therapy } b, d\end{array}$ & $7.0-11.0$ & 164 \\
\hline Total number of patients, $N$ & & & 4638 \\
\hline
\end{tabular}

$A 1 C$ glycated hemoglobin, BID twice daily, INS insulin, MET metformin, $S U$ sulfonylurea, TZD thiazolidinedione

a All trials were 24 weeks in duration and the dose of saxagliptin dose was $5 \mathrm{mg} / \mathrm{d}$ unless otherwise noted

b Trial duration was 12 weeks

c Saxagliptin dose was $2.5 \mathrm{mg}$ BID

d Saxagliptin dose was $2.5 \mathrm{mg} / \mathrm{d}$

of $<7$ and $\leq 6.5 \%$ (expressed as percentages) for saxagliptin and control were determined using RevMan. Between-treatment differences in study endpoints were analyzed using a random effects method where the weight assigned to each study was the inverse of the variance of the effect estimate. Thus, in general, larger studies were given more weight than smaller studies.

\section{RESULTS}

\section{Study Characteristics}

This analysis included 14 phase 2 or 3 studies (Table 1) that evaluated saxagliptin $5 \mathrm{mg} / \mathrm{d}$ or control as either monotherapy $(n=1196 ; 5$ studies), add-on therapy ( $n=2139$ vs placebo and $n=514$ vs uptitrated sulfonylurea; 6 studies and 1 study, respectively), or initial combination therapy ( $n=619$ vs control monotherapy; 1 study); or, for patients with renal impairment, saxagliptin $2.5 \mathrm{mg} / \mathrm{d}$ or placebo $(n=164 ; 1$ study). All studies were 12 or 24 weeks in duration. Data from all 14 trials were included in the analysis of change from baseline in A1C; availability of data per study varied for all other end points.

Mean (SD) baseline A1C levels for saxagliptin and control were $8.12 \%(0.9 \%)$ and $8.07 \%$ $(0.9 \%)$ for monotherapy versus placebo, $8.26 \%$ $(1.0 \%)$ and $8.15 \%(0.9 \%)$ for add-on versus placebo, $8.26 \%(0.8 \%)$ and $8.15 \%(0.9 \%)$ for add-on versus uptitrated sulfonylurea, $9.41 \%$ 
(1.3\%) and 9.43\% (1.3\%) for initial combination therapy versus control monotherapy, and $8.45 \%(1.2 \%)$ and $8.09 \%(1.1 \%)$ for saxagliptin versus placebo in patients with renal impairment (Fig. 1).

\section{Glycemic Outcomes}

For all 14 studies combined, the reduction from baseline in A1C was greater with saxagliptin versus control (mean treatment difference [95\% CI] $-0.55 \% \quad[-0.63 \%,-0.47 \%]$; Fig. 1). Large reductions in A1C were observed whether saxagliptin was used as a monotherapy versus placebo $(-0.52 \%[-0.63 \%,-0.40 \%] ; 5$ studies $)$ or as an add-on versus placebo $(-0.55 \%$ [ $-0.69 \%$, $-0.40 \%] ; 6$ studies). Marked reductions in A1C from baseline were also observed whether saxagliptin was used as an add-on versus uptitrated sulfonylurea $\quad(-0.72 \% \quad[-0.88 \%,-0.56 \%] ; 1$ study) or as an initial combination therapy versus control monotherapy $(-0.54 \%[-0.73 \%$, $-0.35 \%] ; 1$ study). Large reductions in A1C with saxagliptin versus placebo were also noted in patients with renal impairment $(-0.42 \%$ $[-0.75 \%,-0.09 \%] ; 1$ study). Similar treatment effects were observed across subgroups of age, sex, and geographical region (Figs. S1-S3 in the Electronic supplementary material, ESM). Reductions from baseline in A1C favored saxagliptin versus control for patients $<65$ years of age $(-0.55 \% \quad[-0.67 \%,-0.43 \%] ; 9$ studies $)$, patients $\geq 65$ years of age $(0.54 \% \quad[-0.69 \%$ $[-0.69 \%,-0.38 \%] ; 10$ studies), men $(-0.54 \%$ $[-0.69 \%,-0.40 \%] ; 10$ studies), and women (-0.55\% [-0.64\%, $-0.47 \%] ; 10$ studies). The A1C reduction from baseline was generally similar across geographical regions of North America, Europe, Latin America, and Asia. A smaller reduction from baseline in $\mathrm{A} 1 \mathrm{C}$ was observed

\begin{tabular}{|c|c|c|c|c|c|c|c|c|}
\hline Study & $\begin{array}{c}\text { SAXA, } 5 \text { mg } \\
\text { Mean } \\
\text { baseline } \\
\text { A1C, } \%\end{array}$ & \multicolumn{2}{|c|}{$\begin{array}{l}\text { SAXA, } 5 \mathrm{mg} \\
\text { Mean change } \\
\text { from baseline }\end{array}$} & $\begin{array}{c}\text { CTL } \\
\text { Mean } \\
\text { baseline } \\
\text { A1C, \% }\end{array}$ & \multicolumn{2}{|c|}{$\begin{array}{c}\text { CTL } \\
\text { Mean change } \\
\text { from baseline }\end{array}$} & $\begin{array}{c}\text { Mean treatment } \\
\text { difference in change } \\
\text { from baseline (A1C, \%) } \\
\text { IV, Random, } 95 \% \mathrm{Cl}\end{array}$ & $\begin{array}{c}\text { Mean treatment } \\
\text { difference in change } \\
\text { from baseline (A1C, \% } \\
\text { IV, Random, } 95 \% \mathrm{Cl}\end{array}$ \\
\hline Monotherapy (CV181008[35]) & 8.05 & $-0.90(0.91)$ & 42 & 7.92 & $-0.27(0.87)$ & 62 & $\longrightarrow$ & $-0.63(-0.98,-0.28)$ \\
\hline Monotherapy (CV181011[24]) & 7.98 & $-0.46(1.01)$ & 103 & 7.88 & $0.19(0.96)$ & 92 & & $-0.65(-0.93,-0.37)$ \\
\hline Monotherapy (CV181038[23]) & 7.93 & $-0.66(0.85)$ & 69 & 7.79 & $-0.26(0.85)$ & 68 & & $-0.40(-0.68,-0.12)$ \\
\hline Monotherapy (CV181063[36]) & 8.15 & $-0.84(1.12)$ & 277 & 8.14 & $-0.34(1.08)$ & 274 & $\rightarrow$ & $-0.50(-0.68,-0.32)$ \\
\hline Monotherapy (CV181082[37]) & 8.33 & $-0.51(1.01)$ & 104 & 8.33 & $-0.05(1.00)$ & 105 & $\rightarrow$ & $-0.46(-0.73,-0.19)$ \\
\hline Overall pooled & 8.12 & & 595 & 8.07 & & 601 & $\bullet$ & $-0.52(-0.63,-0.40)$ \\
\hline Add-on to INS+MET (CV181057[38]) & 8]) 8.67 & $-0.73(0.94)$ & 300 & 8.66 & $-0.32(0.90)$ & 149 & 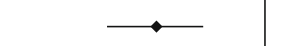 & $-0.41(-0.59,-0.23)$ \\
\hline Add-on to MET (CV181014[39]) & 8.07 & $-0.69(0.95)$ & 186 & 8.06 & $0.13(0.94)$ & 175 & & $-0.82(-1.02,-0.62)$ \\
\hline Add-on to MET (CV181080[40]) & 7.92 & $-0.56(0.77)$ & 74 & 7.97 & $-0.22(0.77)$ & 84 & 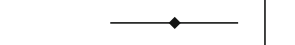 & $-0.34(-0.58,-0.10)$ \\
\hline Add-on to MET (CV181064[41]) & 7.90 & $-0.78(0.85)$ & 275 & 7.94 & $-0.37(0.84)$ & 279 & $\longrightarrow$ & $-0.41(-0.55,-0.27)$ \\
\hline Add-on to MET+SU (CV181117[26]) & ]) 8.37 & $-0.74(0.85)$ & 127 & 8.17 & $-0.08(0.83)$ & 127 & $\cdot$ & $-0.66(-0.87,-0.45)$ \\
\hline Add-on to TZD (CV181013[27]) & 8.35 & $-0.94(1.01)$ & 183 & 8.19 & $-0.30(1.02)$ & 180 & $\longrightarrow$ & $-0.64(-0.85,-0.43)$ \\
\hline Overall pooled & 8.26 & & 1145 & 8.15 & & 994 & $\diamond$ & $-0.55(-0.69,-0.40)$ \\
\hline Add-on vs SU (CV181040[42]) & 8.48 & $-0.64(0.93)$ & 250 & 8.44 & $0.08(0.93)$ & 264 & 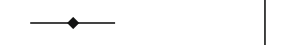 & $-0.72(-0.88,-0.56)$ \\
\hline $\begin{array}{l}\text { Initial combo vs MET } \\
\text { monotherapy (CV181039[28]) }\end{array}$ & 9.41 & $-2.53(1.22)$ & 306 & 9.43 & $-1.99(1.22)$ & 313 & 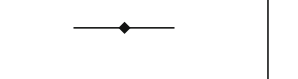 & $-0.54(-0.73,-0.35)$ \\
\hline Renal impairment (CV181062[43]) & 8.45 & $-0.86(1.03)$ & 81 & 8.09 & $-0.44(1.11)$ & 83 & & $-0.42(-0.75,-0.09)$ \\
\hline \multirow[t]{2}{*}{ Total } & & & 2377 & & & 2255 & $\diamond$ & $-0.55(-0.63,-0.47)$ \\
\hline & & & & & & & $\begin{array}{l}-0.5 \\
\text { Favors SAXA }\end{array}$ & $\begin{array}{c}0.5 \\
\text { Favors CTL }\end{array}$ \\
\hline
\end{tabular}

Fig. 1 Mean treatment difference in change in A1C from baseline across treatment regimens. $A 1 C$ glycated hemoglobin, $C T L$ control, $I N S$ insulin, $I V$ inverse variance, $M E T$ metformin, $S A X A$ saxagliptin, $S U$ sulfonylurea, $T Z D$ thiazolidinedione 

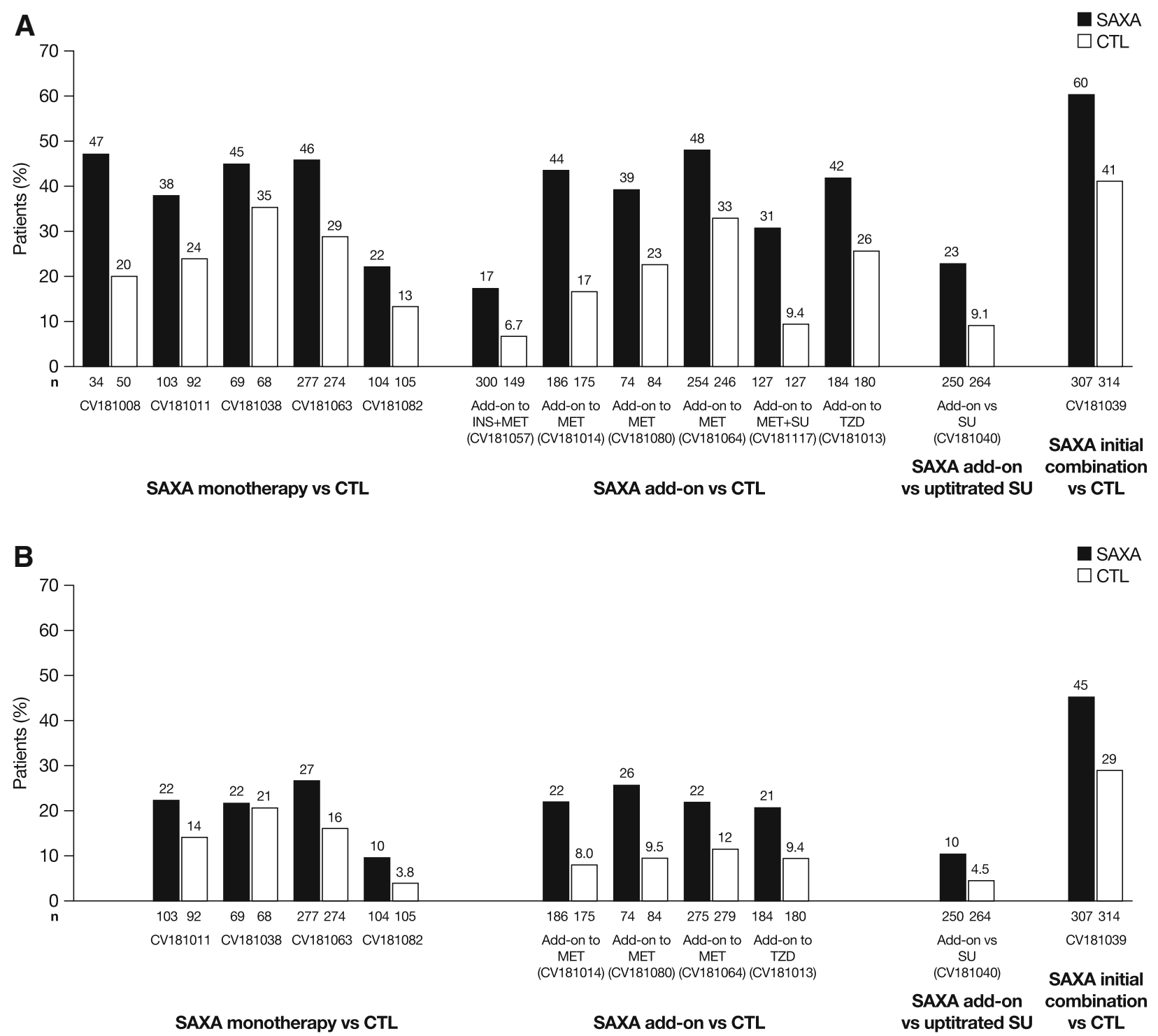

Fig. 2 Percentages of patients who achieved a A1C $<7 \%$ and $\mathbf{b} \mathrm{A} 1 \mathrm{C} \leq 6.5 \%$. $C T L$ control, INS insulin, $I V$ inverse variance, $M E T$ metformin, $S A X A$ saxagliptin, $S U$ sulfonylurea, $T Z D$ thiazolidinedione

for Africa; however, data were limited to a single add-on to insulin trial.

Across the combined studies, patients treated with saxagliptin were more likely to achieve A1C $<7 \%$ (39\% vs 23\%; treatment difference [95\% CI] $0.16[0.13,0.19] ; 13$ studies) and A1C $\leq 6.5 \%$ (24\% vs $14 \%$; treatment difference [95\% $\mathrm{CI}] 0.10$ [0.07, 0.13]; 10 studies) versus control, with similar effects obtained when saxagliptin was used as a monotherapy or add-on therapy (Fig. $2 \mathrm{a}, \mathrm{b}$ ). The percentage of patients achieving $\mathrm{A} 1 \mathrm{C}<7 \%$ was greater with saxagliptin whether it was used as a monotherapy versus placebo ( $40 \%$ vs $25 \%$ ), an add-on versus placebo (36\% vs $21 \%$ ), an add-on versus uptitrated sulfonylurea (23\% vs $9.1 \%$ ), or as initial combination therapy versus control monotherapy (60\% vs $41 \%)$. Similarly, the percentage of patients achieving $\mathrm{A} 1 \mathrm{C} \leq 6.5 \%$ was greater with saxagliptin monotherapy versus placebo $(22 \%$ vs $14 \%)$, add-on versus placebo ( $22 \%$ vs $10 \%)$, add-on versus uptitrated sulfonylurea (10\% vs $4.5 \%)$, and initial combination therapy versus control monotherapy ( $45 \%$ vs $29 \%$ ). 
Table 2 Effect estimates for mean treatment differences in glycemic efficacy end-point changes from baseline for saxagliptin versus control in adults with T2D

\begin{tabular}{|c|c|c|c|c|c|c|c|}
\hline Outcome & $\begin{array}{l}\text { Baseline } \\
\text { saxagliptin }\end{array}$ & $\begin{array}{l}\text { Saxagliptin, } \\
n\end{array}$ & $\begin{array}{l}\text { Baseline } \\
\text { control }\end{array}$ & $\begin{array}{l}\text { Control, } \\
n\end{array}$ & $\begin{array}{l}\text { Studies, } \\
n\end{array}$ & $\begin{array}{l}\text { Mean treatment } \\
\text { difference }^{\mathrm{a}} \mathrm{IV} \text {, } \\
\text { random }\end{array}$ & 95\% CI \\
\hline FPG, mg/dL & 170.65 & 2319 & 170.09 & 2188 & 13 & -12.89 & $-15.82,-9.97$ \\
\hline $\begin{array}{l}\text { PPG AUC, } \\
\mathrm{g} \min / \mathrm{dL}\end{array}$ & 46,370 & 1235 & 46,684 & 1068 & 10 & -4.67 & $-6.03,-3.30$ \\
\hline $\begin{array}{l}\text { Fasting insulin, } \\
\mathrm{uU} / \mathrm{mL}\end{array}$ & 11.74 & 1867 & 11.66 & 1839 & 11 & -0.15 & $-0.65,0.35$ \\
\hline $\begin{array}{l}\text { Insulin AUC, } \\
\mathrm{mU} \mathrm{min} / \mathrm{mL}\end{array}$ & 5576.7 & 838 & 5783.2 & 809 & 8 & 1.14 & $0.65,1.63$ \\
\hline $\begin{array}{l}\text { Fasting C-peptide, } \\
\mathrm{ng} / \mathrm{mL}\end{array}$ & 2.87 & 1859 & 2.92 & 1708 & 10 & -0.01 & $-0.09,0.06$ \\
\hline $\begin{array}{l}\text { C-peptide AUC, } \\
\text { ng } \min / \mathrm{mL}\end{array}$ & 936.5 & 942 & 936.9 & 822 & 8 & 82.23 & $33.73,130.74$ \\
\hline Glucagon, $\mathrm{pg} / \mathrm{mL}$ & 73.66 & 1747 & 71.65 & 1597 & 10 & -2.22 & $-3.92,-0.52$ \\
\hline $\begin{array}{c}\text { Glucagon AUC, } \\
\text { ng } \min / \mathrm{mL}\end{array}$ & 14,896 & 1035 & 14,564 & 860 & 8 & -0.93 & $-1.28,-0.58$ \\
\hline HOMA- $2 \% \beta, \%$ & 61.41 & 1699 & 60.78 & 1640 & 10 & 7.55 & $5.32,9.78$ \\
\hline HOMA-2 IR, \% & 3.17 & 1109 & 3.18 & 1099 & 7 & -0.14 & $-0.24,-0.04$ \\
\hline
\end{tabular}

$A U C$ area under the curve, FPG fasting plasma glucose, $H O M A$ homeostasis model assessment of $\beta$-cell function, $I R$ insulin resistance, $I V$ inverse variance, $P P G$ postprandial glucose; T2D type 2 diabetes

a Mean treatment difference in change from baseline for saxagliptin versus control

The reduction from baseline in FPG was greater with saxagliptin versus control across the combined studies (mean treatment difference $[95 \% \mathrm{CI}]-12.89 \mathrm{mg} / \mathrm{dL}[-15.82,-9.97] ; 13$ studies, Table 2). Likewise, a greater reduction from baseline in PPG AUC was observed with saxagliptin versus control across 10 studies (mean treatment difference $[95 \%$ CI $]$ $-4.67 \mathrm{~g}$ min/dL [-6.03, -3.30]; Table 2).

No difference was observed for change from baseline in fasting insulin between saxagliptin versus control (mean treatment difference [95\% $\mathrm{CI}]-0.15 \mu \mathrm{U} / \mathrm{mL}[-0.65,0.35] ; 11$ studies), whereas there was a modest increase from baseline in insulin AUC with saxagliptin versus control (mean treatment difference [95\% CI] $1.14 \mathrm{mU} \mathrm{min} / \mathrm{mL} \quad[0.65,1.63] ; 8$ studies, Table 2). Results for C-peptide paralleled those observed for insulin, with no clinically meaningful differences observed for change from baseline in fasting C-peptide and an increase from baseline in C-peptide AUC with saxagliptin versus control.

The reduction from baseline in fasting glucagon was greater with saxagliptin versus placebo across 10 studies combined (mean treatment difference $\left[\begin{array}{lll}95 \% & \mathrm{CI}\end{array}\right]-2.22 \mathrm{pg} / \mathrm{mL}$ $[-3.92,-0.52])$. Similarly, a greater reduction from baseline in glucagon AUC was observed with saxagliptin versus control across eight studies (mean treatment difference [95\% CI] $-0.93 \mathrm{ng} \mathrm{min} / \mathrm{mL}[-1.28,-0.58]$, Table 2).

$\beta$-Cell function, calculated via the change from baseline in HOMA- $2 \% \beta$, improved with saxagliptin versus control (mean treatment difference $[95 \% \mathrm{CI}] \quad 7.55 \% \quad[5.32 \%, 9.78 \%] ; 10$ studies). The change from baseline in HOMA-2 IR indicated a slight reduction in insulin 
Table 3 Total effect estimates for mean treatment differences in weight and blood pressure end-point changes from baseline for saxagliptin versus control in adults with T2D

\begin{tabular}{llllllll}
\hline Parameter & $\begin{array}{l}\text { Baseline } \\
\text { saxagliptin }\end{array}$ & $\begin{array}{l}\text { Saxagliptin, } \\
\boldsymbol{n}\end{array}$ & $\begin{array}{l}\text { Baseline } \\
\text { control }\end{array}$ & $\begin{array}{l}\text { Control, } \\
\boldsymbol{n}\end{array}$ & $\begin{array}{l}\text { Studies, } \\
\boldsymbol{n}\end{array}$ & $\begin{array}{l}\text { Mean treatment } \\
\text { difference }^{\mathbf{a}} \text { IV, random }\end{array}$ & 95\% CI \\
\hline $\begin{array}{l}\text { Body weight, kg } \\
\text { Waist }\end{array}$ & 79.64 & 2195 & 78.93 & 2064 & 12 & 0.39 & $0.14,0.64$ \\
$\quad \begin{array}{l}\text { circumference, } \\
\text { cm }\end{array}$ & 97.06 & 1720 & 97.93 & 1727 & 11 & 0.31 & $-0.06,0.69$ \\
$\begin{array}{l}\text { BMI, kg/m } \\
\text { B }\end{array}$ & 29.31 & 2121 & 29.03 & 1980 & 11 & 0.17 & \\
$\begin{array}{l}\text { Blood pressure } \\
\text { Systolic, mmHg }\end{array}$ & 128.8 & 1934 & 129.1 & 1720 & 14 & -0.19 & $0.06,0.28$ \\
$\begin{array}{l}\text { Diastolic, } \\
\text { mmHg }\end{array}$ & 79.3 & 1934 & 79.4 & 1720 & 14 & 0.09 & $-1.07,0.68$ \\
\hline
\end{tabular}

$I V$ inverse variance, $T 2 D$ type 2 diabetes

${ }^{a}$ Mean treatment difference in change from baseline for saxagliptin versus control

Table 4 Total effect estimates for mean treatment differences in lipid end-point percentage changes from baseline for saxagliptin versus control in adults with T2D

\begin{tabular}{|c|c|c|c|c|c|c|c|}
\hline Parameter & $\begin{array}{l}\text { Baseline } \\
\text { saxagliptin }\end{array}$ & $\begin{array}{l}\text { Saxagliptin, } \\
n\end{array}$ & $\begin{array}{l}\text { Baseline } \\
\text { control }\end{array}$ & $\begin{array}{l}\text { Control, } \\
n\end{array}$ & $\begin{array}{l}\text { Studies, } \\
n\end{array}$ & $\begin{array}{l}\text { Mean treatment } \\
\text { difference }^{\mathrm{a}} \text { IV, } \\
\text { random }\end{array}$ & 95\% CI \\
\hline $\begin{array}{l}\text { Total cholesterol, } \\
\mathrm{mg} / \mathrm{dL}\end{array}$ & 194.65 & 1617 & 194.41 & 1616 & 10 & -1.47 & $\begin{array}{l}-2.62 \\
-0.33\end{array}$ \\
\hline $\begin{array}{l}\text { LDL cholesterol, } \\
\mathrm{mg} / \mathrm{dL}\end{array}$ & 112.98 & 1471 & 112.88 & 1473 & 10 & -0.73 & $\begin{array}{r}-2.75 \\
1.29\end{array}$ \\
\hline $\begin{array}{l}\text { HDL cholesterol, } \\
\mathrm{mg} / \mathrm{dL}\end{array}$ & 45.36 & 1616 & 44.71 & 1616 & 10 & -1.74 & $\begin{array}{l}-2.92 \\
-0.57\end{array}$ \\
\hline $\begin{array}{l}\text { Fasting } \\
\text { triglycerides, } \\
\mathrm{mg} / \mathrm{dL}\end{array}$ & 192.75 & 1617 & 201.80 & 1615 & 10 & -4.08 & $\begin{array}{l}-7.30 \\
-0.85\end{array}$ \\
\hline $\begin{array}{l}\text { Free fatty acids, } \\
\mathrm{mg} / \mathrm{dL}\end{array}$ & 0.53 & 957 & 0.54 & 962 & 7 & -2.53 & $\begin{array}{r}-8.19 \\
3.13\end{array}$ \\
\hline
\end{tabular}

$H D L$ high-density lipoprotein, $I V$ inverse variance, $L D L$ low-density lipoprotein, $T 2 D$ type-2 diabetes

${ }^{a}$ Mean treatment difference in percentage change from baseline for saxagliptin versus control 
resistance with saxagliptin versus control (mean treatment difference $[95 \% \mathrm{CI}]-0.14 \%[-0.24 \%$, $-0.04 \%] ; 7$ studies, Table 2).

\section{Metabolic (Weight, Blood Pressure, and Lipid) Outcomes}

No clinically meaningful effects were observed for change from baseline in body weight (mean treatment difference [95\% CI] 0.39 [0.14, 0.64] $\mathrm{kg} ; 12$ studies), waist circumference (0.31 $[-0.06,0.69] \mathrm{cm} ; 11$ studies), or BMI (0.17 [0.06, $0.28] \mathrm{kg} / \mathrm{m}^{2} ; 11$ studies) with saxagliptin versus control (Table 3). Changes from baseline in systolic (mean treatment difference [95\% CI] -0.19 [-1.07, 0.68] mmHg; 14 studies) and diastolic $(0.09[-0.45,0.64] \mathrm{mmHg} ; 14$ studies $)$ blood pressure were similar with saxagliptin and control (Table 3).

Modest reductions in percentage change from baseline in total cholesterol (mean treatment difference $[95 \% \mathrm{CI}]-1.47 \%[-2.62 \%$, $-0.33 \%] ; 10$ studies), HDL cholesterol $(-1.74 \%$, $-2.92 \%,-0.57 \%] ; 10$ studies), and fasting triglycerides $(-4.08 \%[-7.30 \%$ to $-0.85 \%] ; 10$ studies) were observed, along with tendencies for small reductions in LDL cholesterol $(-0.73 \%$ [-2.75\% to $1.29 \%] ; 10$ studies) and free fatty acid $(-2.53 \%$ [-8.19\% to $3.13 \%] ; 7$ studies) with saxagliptin versus control (Table 4).

\section{DISCUSSION}

These analyses demonstrate that the efficacy of saxagliptin is consistent, whether given as a monotherapy, add-on therapy, or initial combination therapy. The effects are likewise consistent in patients with renal impairment, regardless of age and sex, and in different geographic regions. Patients with T2D were more likely to achieve therapeutic glycemic goals of either $\mathrm{A} 1 \mathrm{C}<7 \%$ or $\leq 6.5 \%$ with saxagliptin than with controls. Patients experienced greater mean reductions in A1C, FPG, PPG, and glucagon, as well as greater mean increases in postprandial insulin secretion and C-peptide levels. There were improvements in $\beta$-cell function as assessed by HOMA-2\% $\beta$.
Saxagliptin prevents the degradation of incretins by inhibiting DPP-4. Incretins play a major role in glucose control, with as much as $70 \%$ of postprandial insulin secretion mediated by the incretins GIP and GLP-1 [44]. After eating, patients experience a rapid and transient increase in GIP and GLP-1, with degradation by DPP-4 within minutes of secretion [45-47]. Saxagliptin at doses of $2.5-400 \mathrm{mg}$ inhibits plasma DPP-4 activity by $50-79 \%$ at $24 \mathrm{~h}$ [48]. Consequently, at the recommended dose of $5 \mathrm{mg} / \mathrm{d}$ in patients with T2D, GIP and GLP-1 concentrations are at least twofold more than baseline $[48,49]$. The incretin effects of saxagliptin have been demonstrated as improvements in FPG, PPG, and glucose disposal through a combination of increased fasting and postprandial insulin secretion and a reduction in postprandial glucagon secretion in patients with T2D [49]. Our analysis demonstrated an increase in insulin AUC and C-peptide AUC and reductions in fasting and postprandial glucagon secretion. Although we did not see an increase in fasting insulin or fasting C-peptide, treatment with saxagliptin was associated with significant reductions in both FPG and PPG.

In addition to improving glucoregulatory hormonal secretion, DPP-4 inhibition may also be associated with protective and/or restorative effects on $\beta$-cell function. Preclinical studies using a high-fat diet-fed, streptozotocin-induced mouse model of diabetes have shown that saxagliptin has beneficial effects on $\beta$-cell mass and islet cell morphology [50], with clinical studies also demonstrating small positive effects on $\beta$-cell function. After a median 2-year follow-up, treatment with saxagliptin was associated with preservation of $\beta$-cell function, assessed by HOMA-2\% $\beta$, whereas treatment with placebo was associated with a decline in $\beta$-cell function in patients with a history of T2D and CVD who participated in the SAVOR-TIMI 53 trial $(N=16,492)[19,51]$. These positive effects on pancreatic $\beta$-cells may explain the small improvements in HOMA- $2 \% \beta$ observed in this study.

The results presented here are similar to a meta-analysis of 16 randomized, controlled studies $\geq 12$ weeks in duration that examined the efficacy of DPP-4 inhibitors, including 
saxagliptin, compared with placebo in adults with T2D [52]. The reduction from baseline in A1C was greater with DPP-4 inhibitors versus placebo (weighted mean difference [95\% CI] $-0.74 \% \quad[-0.85 \%,-0.62 \%] ; 16$ studies), with similar efficacies observed when used as a monotherapy or add-on therapy [52]. The magnitude of $\mathrm{A} 1 \mathrm{C}$ reduction in the current analysis with saxagliptin $(-0.55 \%$ [ $-0.63 \%$, $-0.47 \%]$ ) was slightly lower than that obtained with either vildagliptin $(-0.73 \% \quad[-0.94 \%$, $-0.52 \%] ; 9$ studies) or sitagliptin $(-0.74 \%$ $[-0.84 \%,-0.63 \%] ; 7$ studies), but this may be explained by differences in clinical study design, such as baseline A1C, T2D duration, concomitant glucose-lowering agents, study duration, run-in period, and washout [52]. Similar to the analyses reported here for saxagliptin, a greater percentage of patients achieved $\mathrm{A} 1 \mathrm{C}<7 \%$ (43\% vs $17 \%$; 9 studies) and there was a greater reduction in FPG (weighted mean difference, $-18[-22,-14] \mathrm{mg} / \mathrm{dL} ; 15$ studies) with DPP-4 inhibitors versus placebo. Neutral effects of DPP-4 inhibitors versus placebo on nonglycemic parameters such as body weight and lipids were also observed [52]. These neutral effects on weight and lipids are consistent with our meta-analysis. Weight neutrality is a desirable attribute for a glucose-lowering agent, given the association of obesity with CVD morbidity and mortality.

In addition to efficacy, the safety and risk-benefit profiles of glucose-lowering agents are important considerations when selecting appropriate therapies for patients. This report does not include a safety analysis because a previously published pooled analysis of 20 randomized controlled studies, including 13 of the studies in the current meta-analysis, demonstrated the safety and tolerability of saxagliptin when used as a monotherapy or add-on therapy [34]. Analysis of these 20 studies found that the incidence rates (per 100 patient-years) of serious adverse events (saxagliptin, 7.3; control, 7.2) and discontinuations due to adverse events (saxagliptin, 4.2; control, 3.9) with saxigliptin were similar to those obtained with the control, and were consistent irrespective of age and sex.
Compared with the controls, premature discontinuation rates with saxagliptin were similar in eight studies, higher in three studies, and lower in nine studies [34].

The limitations of our analyses are the small number of studies in some treatment regimens and the inclusion of only a single study for some treatment regimen categories. Because these analyses were limited to studies 12 or 24 weeks in duration, the durability of the efficacy of saxagliptin could not be evaluated. However, the long-term efficacy of saxagliptin was demonstrated previously [53]. Although we recognize these limitations, our study has several strengths. Its large size is representative of a heterogeneous patient population, and the included studies were randomized, double-blind, placebo- or active-controlled studies. The inclusion of a broad patient population allowed for subgroup analysis, which demonstrated the efficacy of saxagliptin across subgroups defined by age, sex, or geographical location.

\section{CONCLUSION}

Improving glycemic control is of the utmost importance when attempting to slow the progression of $\mathrm{T} 2 \mathrm{D}$ and prevent microvascular complications. Thus, glucose-lowering agents such as saxagliptin with glycemic efficacy, a low risk for hypoglycemia, and neutral effects on metabolic end points are advantageous. The consistency of saxagliptin efficacy in different patient subgroups with T2D and across treatment regimens, as shown here, demonstrates its usefulness in a broad range of treatment settings.

\section{ACKNOWLEDGEMENTS}

Sponsorship for this study and article processing charges were funded by AstraZeneca. All named authors meet the International Committee of Medical Journal Editors (ICMJE) criteria for authorship of this manuscript and gave final approval to the version to be published. All 
authors had full access to all of the data in this study and take complete responsibility for the integrity of the data and accuracy of the data analysis. Medical writing support for the preparation of this manuscript was provided by Lauren D'Angelo, PhD, and Janet Matsuura, $\mathrm{PhD}$, from Complete Healthcare Communications, LLC (Chadds Ford, PA, USA), with funding from AstraZeneca.

Disclosures. Mikaela Sjöstrand is an employee of AstraZeneca. Cheryl Wei is an employee of AstraZeneca. William Cook is an employee of MedImmune, a subsidiary of AstraZeneca. Kristina Johnsson is an employee of AstraZeneca. Pia S. Pollack was an employee of AstraZeneca at the time this research was conducted and owns AstraZeneca stock. Christina Stahre is an employee of AstraZeneca. Boaz Hirshberg is an employee of MedImmune, a subsidiary of AstraZeneca.

Compliance with Ethics Guidelines. This article does not contain any new studies with human subjects performed by any of the authors. In the studies included in this meta-analysis, all procedures followed were in accordance with the ethical standards of the responsible committee on human experimentation (institutional and national) and with the Declaration of Helsinki (1964). Informed consent was obtained from all patients before they were included in the studies.

Data Availability. The datasets generated and/or analyzed during the current study are available from the corresponding author on reasonable request.

Open Access. This article is distributed under the terms of the Creative Commons Attribution-NonCommercial 4.0 International License (http://creativecommons.org/licenses/ by-nc/4.0/), which permits any noncommercial use, distribution, and reproduction in any medium, provided you give appropriate credit to the original author(s) and the source, provide a link to the Creative Commons license, and indicate if changes were made.

\section{REFERENCES}

1. Menke A, Casagrande S, Geiss L, Cowie CC. Prevalence of and trends in diabetes among adults in the United States, 1988-2012. JAMA. 2015;314:1021-9.

2. Guariguata L, Whiting DR, Hambleton I, Beagley J, Linnenkamp U, Shaw JE. Global estimates of diabetes prevalence for 2013 and projections for 2035 . Diabetes Res Clin Pract. 2014;103:137-49.

3. International Diabetes Federation. IDF diabetes atlas, sixth edition. 2014 [cited 2016 September 27]. Available from: http://www.idf.org/diabetesatlas/ 5e/the-global-burden.

4. Fonseca VA. Defining and characterizing the progression of type 2 diabetes. Diabetes Care. 2009;32:S151-6.

5. American Diabetes Association. Approaches to glycemic treatment. Diabetes Care. 2016;39:S52-9.

6. Garber AJ, Abrahamson MJ, Barzilay JI, et al. Consensus statement by the American Association of Clinical Endocrinologists and American College of Endocrinology on the comprehensive type 2 diabetes management algorithm-2016 executive summary. Endocr Pract. 2016;22:84-113.

7. Mozaffarian D, Benjamin EJ, Go AS, et al. Heart disease and stroke statistics-2016 update: a report from the American Heart Association. Circulation. 2016;133:e38-360.

8. Morrish NJ, Wang SL, Stevens LK, Fuller JH, Keen H. Mortality and causes of death in the WHO Multinational Study of Vascular Disease in Diabetes. Diabetologia. 2001;44:S14-21.

9. Stark Casagrande S, Fradkin JE, Saydah SH, Rust KF, Cowie CC. The prevalence of meeting A1C, blood pressure, and LDL goals among people with diabetes, 1988-2010. Diabetes Care. 2013;36:2271-9.

10. UK Prospective Diabetes Study (UKPDS) Group. Intensive blood-glucose control with sulphonylureas or insulin compared with conventional treatment and risk of complications in patients with type 2 diabetes. Lancet. 1998;352:837-53.

11. Holman RR, Paul SK, Bethel MA, Matthews DR, Neil HA. 10-Year follow-up of intensive glucose control in type 2 diabetes. N Engl J Med. 2008;359:1577-89.

12. Hayward RA, Reaven PD, Wiitala WL, et al. Follow-up of glycemic control and cardiovascular outcomes in type 2 diabetes. $\mathrm{N}$ Engl J Med. 2015;372:2197-206. 
13. Zinman B, Wanner C, Lachin JM, et al. Empagliflozin, cardiovascular outcomes, and mortality in type 2 diabetes. N Engl J Med. 2015;373:2117-28.

14. Marso SP, Daniels GH, Brown-Frandsen K, et al. Liraglutide and cardiovascular outcomes in type 2 diabetes. N Engl J Med. 2016;375:311-22.

15. Marso SP, Bain SC, Consoli A, et al. Semaglutide and cardiovascular outcomes in patients with type 2 diabetes. N Engl J Med. 2016;375:1834-1844.

16. Pfeffer MA, Claggett B, Diaz R, et al. Lixisenatide in patients with type 2 diabetes and acute coronary syndrome. N Engl J Med. 2015;373:2247-57.

17. White WB, Cannon CP, Heller SR, et al. Alogliptin after acute coronary syndrome in patients with type 2 diabetes. N Engl J Med. 2013;369:1327-35.

18. Green JB, Bethel MA, Armstrong PW, et al. Effect of sitagliptin on cardiovascular outcomes in type 2 diabetes. N Engl J Med. 2015;373:232-42.

19. Scirica BM, Bhatt DL, Braunwald E, et al. Saxagliptin and cardiovascular outcomes in patients with type 2 diabetes mellitus. $\mathrm{N}$ Engl J Med. 2013;369:1317-26.

20. Zannad F, Cannon C, Cushman WC, et al. Heart failure and mortality outcomes in patients with type 2 diabetes taking alogliptin versus placebo in EXAMINE: a multicentre, randomised, double-blind trial. Lancet. 2015;385:2067-76.

21. AstraZeneca. Onglyza ${ }^{\circledR}$ (saxagliptin): full prescribing information. Wilmington: AstraZeneca; 2016.

22. Drucker DJ. The biology of incretin hormones. Cell Metab. 2006;3:153-65.

23. Frederich R, McNeill R, Berglind N, Fleming D, Chen R. The efficacy and safety of the dipeptidyl peptidase-4 inhibitor saxagliptin in treatment-naive patients with type 2 diabetes mellitus: a randomized controlled trial. Diabetol Metab Syndr. 2012;4:36.

24. Rosenstock J, Aguilar-Salinas C, Klein E, et al. Effect of saxagliptin monotherapy in treatment-naive patients with type 2 diabetes. Curr Med Res Opin. 2009;25:2401-11.

25. DeFronzo RA, Hissa MN, Garber AJ, et al. The efficacy and safety of saxagliptin when added to metformin therapy in patients with inadequately controlled type 2 diabetes with metformin alone. Diabetes Care. 2009;32:1649-55.

26. Moses RG, Kalra S, Brook D, et al. A randomized controlled trial of the efficacy and safety of saxagliptin as add-on therapy in patients with type 2 diabetes and inadequate glycaemic control on metformin plus a sulphonylurea. Diabetes Obes Metab. 2014;16:443-50.

27. Hollander P, Li J, Allen E, Chen R, CV181-013 Investigators. Saxagliptin added to a thiazolidinedione improves glycemic control in patients with type 2 diabetes and inadequate control on thiazolidinedione alone. J Clin Endocrinol Metab. 2009;94:4810-9.

28. Jadzinsky M, Pfutzner A, Paz-Pacheco E, et al. Saxagliptin given in combination with metformin as initial therapy improves glycaemic control in patients with type 2 diabetes compared with either monotherapy: a randomized controlled trial. Diabetes Obes Metab. 2009;11:611-22.

29. Rosenstock J, Hansen L, Zee P, et al. Dual add-on therapy in type 2 diabetes poorly controlled with metformin monotherapy: a randomized double-blind trial of saxagliptin plus dapagliflozin addition versus single addition of saxagliptin or dapagliflozin to metformin. Diabetes Care. 2015;38:376-83.

30. Doucet J, Chacra A, Maheux P, Lu J, Harris S, Rosenstock J. Efficacy and safety of saxagliptin in older patients with type 2 diabetes mellitus. Curr Med Res Opin. 2011;27:863-9.

31. Schernthaner G, Duran-Garcia S, Hanefeld M, et al. Efficacy and tolerability of saxagliptin compared with glimepiride in elderly patients with type 2 diabetes: a randomized, controlled study (GENERATION). Diabetes Obes Metab. 2015;17:630-8.

32. Karyekar CS, Ravichandran S, Allen E, Fleming D, Frederich R. Tolerability and efficacy of glycemic control with saxagliptin in older patients (aged $\geq$ 65 years) with inadequately controlled type 2 diabetes mellitus. Clin Interv Aging. 2013;8:419-30.

33. Iqbal N, Allen E, Ohman P. Long-term safety and tolerability of saxagliptin add-on therapy in older patients (aged $\geq 65$ years) with type 2 diabetes. Clin Interv Aging. 2014;9:1479-87.

34. Hirshberg B, Parker A, Edelberg H, Donovan M, Iqbal N. Safety of saxagliptin: events of special interest in 9156 patients with type 2 diabetes mellitus. Diabetes Metab Res Rev. 2014;30:556-69.

35. Rosenstock J, Sankoh S, List JF. Glucose-lowering activity of the dipeptidyl peptidase- 4 inhibitor saxagliptin in drug-naive patients with type 2 diabetes. Diabetes Obes Metab. 2008;10:376-86.

36. Pan CY, Yang W, Tou C, Gause-Nilsson I, Zhao J. Efficacy and safety of saxagliptin in drug-naive Asian patients with type 2 diabetes mellitus: a randomized controlled trial. Diabetes Metab Res Rev. 2012;28:268-75. 
37. Prasanna Kumar KM, Jain SM, Tou C, Schutzer KM. Saxagliptin as initial therapy in treatment-naive Indian adults with type 2 diabetes mellitus inadequately controlled with diet and exercise alone: a randomized, double-blind, placebo-controlled, phase IIIb clinical study. Int J Diabetes Dev Ctries. 2014;34:201-9.

38. Barnett AH, Charbonnel B, Donovan M, Fleming D. Effect of saxagliptin as add-on therapy in patients with poorly controlled type 2 diabetes on insulin alone or insulin combined with metformin. Curr Med Res Opin. 2012;28:513-23.

39. DeFronzo R, Hissa MN, Garber AJ, et al. Once-daily saxagliptin added to metformin provides sustained glycemic control and is well tolerated over 102 weeks in patients with type 2 diabetes. Diabetes. 2009;58:547.

40. White JL, Buchanan P, Li J, Frederich R. A randomized controlled trial of the efficacy and safety of twice-daily saxagliptin plus metformin combination therapy in patients with type 2 diabetes and inadequate glycemic control on metformin monotherapy. BMC Endocr Disord. 2014;14:17.

41. Yang W, Pan CY, Tou C, Zhao J, Gause-Nilsson I. Efficacy and safety of saxagliptin added to metformin in Asian people with type 2 diabetes mellitus: a randomized controlled trial. Diabetes Res Clin Pract. 2011;94:217-24.

42. Chacra AR, Tan GH, Apanovitch A, Ravichandran S, List J, Chen R. Saxagliptin added to a submaximal dose of sulphonylurea improves glycaemic control compared with uptitration of sulphonylurea in patients with type 2 diabetes: a randomised controlled trial. Int J Clin Pract. 2009;63:1395-406.

43. Nowicki M, Rychlik I, Haller H, Warren L, Suchower L, Gause-Nilsson I. Saxagliptin improves glycaemic control and is well tolerated in patients with type 2 diabetes mellitus and renal impairment. Diabetes Obes Metab. 2011;13:523-32.

44. Nauck M, Stockmann F, Ebert R, Creutzfeldt W. Reduced incretin effect in type 2 (non-insulin-dependent) diabetes. Diabetologia. 1986;29:46-52.

45. Deacon CF, Danielsen P, Klarskov L, Olesen M, Holst JJ. Dipeptidyl peptidase IV inhibition reduces the degradation and clearance of GIP and potentiates its insulinotropic and antihyperglycemic effects in anesthetized pigs. Diabetes. 2001;50:1588-97.

46. Deacon CF, Nauck MA, Meier J, Hucking K, Holst JJ. Degradation of endogenous and exogenous gastric inhibitory polypeptide in healthy and in type 2 diabetic subjects as revealed using a new assay for the intact peptide. J Clin Endocrinol Metab. 2000;85:3575-81.

47. Mentlein R, Gallwitz B, Schmidt WE. Dipeptidyl-peptidase IV hydrolyses gastric inhibitory polypeptide, glucagon-like peptide-1(7-36)amide, peptide histidine methionine and is responsible for their degradation in human serum. Eur J Biochem. 1993;214:829-35.

48. Boulton DW, Geraldes M. Safety, tolerability, pharmacokinetics and pharmacodynamics of once-daily oral doses of saxagliptin for 2 weeks in type 2 diabetic and healthy subjects. Diabetes. 2007;56(suppl 1):606P.

49. Henry RR, Smith SR, Schwartz SL, et al. Effects of saxagliptin on beta-cell stimulation and insulin secretion in patients with type 2 diabetes. Diabetes Obes Metab. 2011;13:850-8.

50. Poucher SM, Cheetham S, Francis J, Zinker B, Kirby M, Vickers SP. Effects of saxagliptin and sitagliptin on glycaemic control and pancreatic beta-cell mass in a streptozotocin-induced mouse model of type 2 diabetes. Diabetes Obes Metab. 2012;14:918-26.

51. Leibowitz G, Cahn A, Bhatt DL, et al. Impact of treatment with saxagliptin on glycaemic stability and $\beta$-cell function in the SAVOR-TIMI 53 study. Diabetes Obes Metab. 2015;17:487-94.

52. Amori RE, Lau J, Pittas AG. Efficacy and safety of incretin therapy in type 2 diabetes: systematic review and meta-analysis. JAMA. 2007;298: 194-206.

53. Rosenstock J, Gross JL, Aguilar-Salinas C, et al. Long-term 4-year safety of saxagliptin in drug-naive and metformin-treated patients with type 2 diabetes. Diabet Med. 2013;30:1472-6. 